\title{
Use of haloperidol versus atypical antipsychotics and risk of in-hospital death in patients with acute myocardial infarction: cohort study
}

\author{
Yoonyoung Park, ${ }^{1,2}$ Brian T Bateman, ${ }^{1,3}$ Dae Hyun Kim, ${ }^{1}$ Sonia Hernandez-Diaz, ${ }^{2}$ \\ Elisabetta Patorno, ${ }^{1}$ Robert J Glynn, ${ }^{1}$ Helen Mogun, ${ }^{1}$ Krista F Huybrechts ${ }^{1}$
}

\begin{abstract}
${ }^{1}$ Division of
Pharmacoepidemiology and Pharmacoeconomics,

Department of Medicine,

Brigham and Women's Hospital and Harvard Medical School,

1620 Tremont Street, Boston,

MA, 02120, USA

${ }^{2}$ Department of Epidemiology, Harvard TH Chan School of

Public Health, Boston, MA, USA

${ }^{3}$ Department of Anesthesiology,

Perioperative and Pain

Medicine, Brigham and

Women's Hospital and Harvard

Medical School, Boston, MA,

USA

Correspondence to: $Y$ Park yypark@mail.harvard.edu

Additional material is published online only. To view please visit the journal online.

Cite this as: BMJ 2018;360:k1218 http://dx.doi.org/10.1136/bmj.k1218

Accepted: 6 March 2018
\end{abstract}

\section{ABSTRACT}

OBJECTIVE

To compare the risk of in-hospital mortality associated with haloperidol compared with atypical antipsychotics in patients admitted to hospital with acute myocardial infarction.

\section{DESIGN}

Cohort study using a healthcare database.

\section{SETTING}

Nationwide sample of patient data from more than 700 hospitals across the United States.

\section{PARTICIPANTS}

6578 medical patients aged more than 18 years who initiated oral haloperidol or oral atypical antipsychotics (olanzapine, quetiapine, risperidone) during a hospital admission with a primary diagnosis of acute myocardial infarction between 2003 and 2014.

\section{MAIN OUTCOME MEASURE}

In-hospital mortality during seven days of follow-up from treatment initiation.

RESULTS

Among 6578 patients (mean age 75.2 years) treated with an oral antipsychotic drug, 1668 (25.4\%) initiated haloperidol and 4910 (74.6\%) initiated atypical antipsychotics. The mean time from admission to start of treatment ( $5.3 \vee 5.6$ days) and length of stay (12.5 $v 13.6$ days) were similar, but

\section{WHAT IS ALREADY KNOWN ON THIS TOPIC}

Studies in outpatients and nursing home residents have consistently suggested an increased risk of death associated with typical antipsychotics compared with atypical antipsychotics

Little evidence exists on the comparative safety of antipsychotics when they are used in hospital to manage symptoms of delirium

Previous randomized controlled trials were underpowered to assess safety endpoints

\section{WHAT THIS STUDY ADDS}

A small increased risk of in-hospital deaths occurred within seven days of initiating oral haloperidol compared with oral atypical antipsychotics among patients with myocardial infarction admitted to hospital

The association was strongest immediately after initiation of haloperidol, and decreased over time; by day 5 the increased risk associated with oral haloperidol was no longer evident

Although haloperidol has long been used as the primary agent to treat patients with delirium, atypical antipsychotics may be less harmful than oral haloperidol for patients with cardiac morbidity who may be more vulnerable to the adverse effects of haloperidol

the mean treatment duration was shorter in patients using haloperidol compared with those using atypical antipsychotics (2.4 $v 3.9$ days). 1:1 propensity score matching was used to adjust for confounding. In intention to treat analyses with the matched cohort, the absolute rate of death per 100 person days was 1.7 for haloperidol (129 deaths) and 1.1 for atypical antipsychotics (92 deaths) during seven days of follow-up from treatment initiation. The survival probability was 0.93 in patients using haloperidol and 0.94 in those using atypical antipsychotics at day 7 , accounting for the loss of follow-up due to hospital discharge. The unadjusted and adjusted hazard ratios of death were 1.51 (95\% confidence interval 1.22 to 1.85 ) and 1.50 (1.14 to 1.96$)$, respectively. The association was strongest during the first four days of follow-up and decreased over time. By day 5, the increased risk was no longer evident (1.12, 0.79 to 1.59). In the as-treated analyses, the unadjusted and adjusted hazard ratios were 1.90 (1.43 to 2.53$)$ and 1.93 (1.34 to 2.76 ), respectively.

\section{CONCLUSION}

The results suggest a small increased risk of death within seven days of initiating haloperidol compared with initiating an atypical antipsychotic in patients with acute myocardial infarction. Although residual confounding cannot be excluded, this finding deserves consideration when haloperidol is used for patients admitted to hospital with cardiac morbidity.

\section{Introduction}

Several studies have compared the safety of typical antipsychotics with atypical antipsychotics when used to treat behavioral symptoms of dementia in elderly people. ${ }^{1-5}$ Although in 2005 and 2008 the US Food and Drug Administration requested inclusion of black box warnings about increased mortality for both classes of antipsychotics, ${ }^{67}$ the FDA stated that evidence at the time was insufficient to conclude whether the risk from typical antipsychotics was greater than that from atypical antipsychotics. Since then, several studies in outpatients or nursing home residents have reported a greater risk of death associated with typical antipsychotics compared with atypical antipsychotics $^{1-5}$ that is unlikely to be explained by bias alone. $^{8}$

The safety of antipsychotics in the inpatient setting, where the most common indication is management of delirium related agitation, ${ }^{910}$ has not been thoroughly evaluated. Currently no drugs have been approved by the FDA for the treatment of delirium, and owing to inconclusive evidence on safety clinical guidelines 
for delirium vary in their recommendation on the choice of antipsychotic. ${ }^{11-13}$ Several randomized controlled trials have reported similar efficacy between haloperidol and atypical antipsychotics in managing symptoms of delirium in patients admitted to hospital. ${ }^{14-18}$ Meta-analyses of these trials showed inconclusive results on safety related endpoints, including mortality, because of insufficient power. ${ }^{19} 20$ In nursing home residents, an increased mortality in haloperidol users compared with risperidone users has been observed within a few days after the initiation of treatment, suggesting an acute effect of haloperidol on the risk of death. ${ }^{1}$ However, atypical antipsychotics can cause orthostatic hypotension, sedation, and anticholinergic side effects that may negatively impact clinical outcomes of patients with delirium. ${ }^{21} \mathrm{~A}$ recent cohort study ${ }^{22}$ suggested that over-sedation or adverse neurologic events might be more common after short term use of atypical antipsychotics compared with typical antipsychotics among patients who underwent cardiac surgery. Furthermore, both typical and atypical antipsychotics can have adverse effects on the cardiovascular system, including QTc prolongation and arrhythmia. ${ }^{1123}$ Therefore, patients admitted to hospital with cardiac morbidity, such as those with an acute coronary syndrome, might be more vulnerable to the adverse effects of antipsychotics. ${ }^{24}$ We investigated the comparative safety of antipsychotics for the treatment of delirium related agitation regarding inhospital mortality in a cohort of patients admitted to hospital with acute myocardial infarction, who are expected to be at high risk of experiencing adverse effects from antipsychotic treatment.

\section{Methods}

\section{Study design and population}

We conducted a cohort study using the Premier Research Database from 2003 to 2014, a national hospital administrative database including data from more than 700 hospitals and representing $20 \%$ of all inpatient discharges in the United States. ${ }^{25}$ It contains charges and date information for drugs, procedures, and diagnostic tests during hospital stays. Diagnostic codes are recorded in the discharge summary for each patient.

The source cohort consisted of patients aged 18 or older admitted to hospital with a primary diagnosis for acute myocardial infarction, identified by ICD-9 codes 410.x0 and 410.x1 (international classification of diseases, ninth revision). Patients were eligible for inclusion in the study if they received one of the four antipsychotics commonly used in hospital-namely, haloperidol, olanzapine, quetiapine, or risperidone. Haloperidol accounted for more than $70 \%$ of all typical antipsychotic use, and chlorpromazine was not considered because it is often used to treat nausea or vomiting in patients admitted to hospital. ${ }^{26}$ Olanzapine, quetiapine, and risperidone together comprised more than $90 \%$ of all atypical antipsychotic use in the study population. Haloperidol is frequently given intravenously but not the atypical antipsychotics, so we focused on oral treatment because of concerns about residual confounding between those who received intravenous haloperidol and those who received oral atypical antipsychotics. To allow us to assess baseline characteristics, we required patients to have a minimum hospital stay of three days with at least two days free of antipsychotic treatment after admission. To exclude prevalent users and to allow for measurement of potential confounders during the baseline period, we required two days of baseline. We also excluded patients who received more than one antipsychotic on the day of treatment initiation, as well as those who had a diagnosis of a mental disorder that is usually treated with antipsychotics (ie, schizophrenia or bipolar disorder). This was done to ensure patients initiated antipsychotics as a treatment for delirium rather than for pre-existing psychiatric conditions. We did not require a recorded diagnosis of delirium since delirium is known to be under-recorded in medical records and the sensitivity of ICD-9 diagnoses can be as low as 3\%. ${ }^{27}$ Instead, we considered the initiation of antipsychotic treatment in the absence of a pre-existing psychiatric diagnosis as an indication of delirium. We excluded patients who received a coronary artery bypass graft because the risk of death in patients with postoperative delirium is likely to differ from that in medically treated patients. Supplementary figure S1 shows how the study cohort was created.

\section{Exposure and outcome}

We defined the exposure as the class of antipsychotic (haloperidol versus atypical antipsychotic) that a patient initiated. Treatment duration was defined based on the number of days from the initiation date (index date) to discontinuation or switching on the basis of prescription information. We considered patients to have discontinued treatment if they had two or more consecutive days without treatment, and we defined switching as either receiving the other class of antipsychotic or receiving non-oral antipsychotics. The study outcome was in-hospital death within seven days of initiating an antipsychotic.

\section{Covariates}

We identified potential confounders that were plausibly associated with both the choice of antipsychotic and the risk of in-hospital death based on clinical knowledge, using information from hospital admission to the day before initiation of an antipsychotic. In addition to hospital characteristics (teaching, urban), the covariates included patient characteristics and conditions that were plausibly associated with the choice of antipsychotic and the risk of in-hospital death (see supplementary table S1 for the list of covariates). Since diagnoses are aggregated in the discharge summary without reference to when they are made, we only included diagnoses of chronic conditions to ensure that the conditions were present at the time of admission and did not develop after initiation of antipsychotic treatment. We also included 
drugs prescribed and procedures received before antipsychotic initiation. We additionally adjusted for number of days from admission to the day before index date, as a longer hospital stay is associated with a worse prognosis. ${ }^{2930}$

\section{Statistical analyses}

We compared the baseline characteristics of the patients who initiated haloperidol with those who initiated an atypical antipsychotic. The primary analysis was an intention to treat analysis with the initial exposure carried forward to the end of the follow-up, based on the hypothesis that the potential effect of antipsychotics begins with the initiation of treatment and causes a cascade of events leading to death. Follow-up started on the index date and lasted until the earliest of death, discharge, or a predefined length of follow-up. In primary analyses, we used a follow-up of seven days because of the short average treatment duration for both groups and the high rate of censoring. For comparison we also present results for follow-up of 30 days. The secondary analyses included three different analytic approaches. Firstly, in the astreated analyses, we followed patients while they were receiving treatment and censored them the day after they discontinued or on the day they switched. Secondly, we conducted a competing risk analysis, because hospital discharge can be seen as a competing risk rather than as a censoring event. We calculated the cumulative incidence function accounting for competing risks non-parametrically and also using the subdistribution hazard model. Lastly, we repeated the main analyses using logistic regression models to see if results are consistent.

We estimated the crude hazard ratio of in-hospital death using Cox proportional hazard regression, and the adjusted hazard ratio was obtained using propensity score matching. In the context of this study, the propensity score is the probability of receiving haloperidol as opposed to one of the three atypical antipsychotics estimated by logistic regression, given the baseline characteristics (see supplementary table $\mathrm{S} 1$ for a complete list). Patients who received an atypical antipsychotic were matched to patients who received haloperidol using a 1:1 nearest neighbor matching algorithm with a caliper of 0.2 of the standard deviation of the propensity score on the logit scale. ${ }^{31}$ Covariate balance between the two groups was assessed after matching, and we considered an absolute standardized difference less than 0.1 as evidence of balance. ${ }^{31} \mathrm{We}$ used robust standard errors to account for the matching in adjusted analyses. ${ }^{32}$ The validity of the proportional hazards assumption was evaluated using a regression model with a time interaction term.

\section{Subgroup and sensitivity analyses}

To examine whether a particular subset of patients is at higher risk, we repeated the intention to treat analysis with seven days of follow-up in subgroups defined by clinical characteristics that are potentially associated with risk of death. Characteristics considered in these exploratory analyses included age $(<75,75-84, \geq 85$, or continuous), degree of comorbidity burden (Charlson comorbidity index score $>4$, or continuous), utilization of an intensive care unit during baseline (yes or no), and the number of antipsychotic treatment days $(\geq 2$ days) because a large proportion of patients received antipsychotics for only one day before switching or discontinuation. We also compared haloperidol with each atypical antipsychotic separately. Propensity scores were re-estimated in all subsets, patients were re-matched, and the likelihood ratio test was used to determine whether a statistically significant interaction existed between subgroup variables and antipsychotic drug class.

For a sensitivity analysis, we included patients who initiated antipsychotics on the first or second day of their hospital stay to examine whether our findings are generalizable to all patients treated with an antipsychotic in hospital. (The likelihood of residual confounding is increased in this analysis owing to potentially incomplete baseline information.) We repeated the main analyses using a conditional Cox model to account for differential censoring owing to the shorter duration of haloperidol treatment. In this analysis, both participants in a matched pair are censored at the time the first one is censored. To examine if the results were consistent with those from the analyses where we used antipsychotic initiation as the proxy of delirium, we also repeated the analyses in the subset of patients who had a recorded diagnosis of delirium. We used a random effects model to acknowledge unmeasured differences in hospital characteristics and practice patterns. Lastly, we excluded patients with a recorded diagnosis of dementia because of the potential for off-label use of antipsychotic treatment outside of hospital for behavioral symptoms of dementia.

Terminal illness in haloperidol users has been suggested as a source of potential residual confounding in outpatient studies. ${ }^{33}$ We therefore examined the patterns of use of medications frequently used in patients who are approaching the end-of-life stage (short acting and long acting opioids, benzodiazepines), ${ }^{34}$ and discontinuation of chronic disease treatments (statins, $\beta$ blockers, angiotensin converting enzyme inhibitors, and angiotensin II receptor blockers) before the index date. ${ }^{35}$

All analyses were conducted using SAS 9.4 (SAS Institute, Cary, NC) and R (R Core Team, 2016). We considered a two sided $\mathrm{P}$ value less than 0.05 to be statistically significant.

\section{Patient involvement}

No patients were involved in setting the research question or the outcome measures, nor were they involved in developing plans for recruitment, design, or implementation of the study. No patients were asked to advise on interpretation or writing up of results. There are no plans to disseminate the results of the research to study participants or the relevant patient community. 


\section{Results}

We identified 1668 patients who received oral haloperidol and 4910 patients who received oral atypical antipsychotics during their hospital admission for acute myocardial infarction between 2003 and 2014. The mean age of the study population was 75.2 years. Patients who initiated haloperidol were older and more likely to be non-white and to receive treatment for a shorter duration than those who initiated atypical antipsychotics (mean $2.4 \mathrm{~V}$ 3.9 days, respectively). Before propensity score matching, patients in the two groups were similar for myocardial infarction type, comorbidity, baseline treatments, time from admission to antipsychotic initiation, and mean length of hospital stay (table 1; see supplementary table S2 for a complete list of the patients' characteristics). The c statistic from the logistic regression model predicting the propensity score was 0.65 , indicating relatively low discrimination. The largely overlapping distributions of propensity scores (see supplementary figure S2) suggest that haloperidol and atypical antipsychotics were used interchangeably in many instances, judged by the measured covariates. We matched $99.5 \%$ of the haloperidol initiators to atypical antipsychotic initiators ( $n=1659)$, and all covariates included in the propensity score were well balanced after matching.
During the first week after treatment initiation, 129 of 1659 haloperidol initiators (7.8\%) and 92 of 1659 atypical antipsychotic initiators (5.5\%) died in the matched cohort. The cumulative incidence of death among haloperidol initiators was consistently higher than among atypical antipsychotic initiators during this time, accounting for competing risks (fig 1). The survival probability at day 7 was 0.93 for haloperidol initiators and 0.94 for atypical antipsychotic initiators. In the intention to treat analysis during follow-up of seven days, the rate of death per 100 person days was 1.7 for haloperidol initiators and 1.1 for atypical antipsychotic initiators (table 2). The crude hazard ratio of death in the unmatched cohort was 1.51 (95\% confidence interval 1.22 to 1.85) and the adjusted hazard ratio in the matched cohort was 1.50 (1.14 to 1.96). In the as-treated analysis (see supplementary table S3), the mean follow-up was 2.8 (SD 1.7) days for haloperidol initiators and 3.7 (2.1) days for atypical antipsychotic initiators. During the seven days' followup, the crude hazard ratio was 1.90 (1.43 to 2.53) and the adjusted hazard ratio was 1.93 (1.34 to 2.76). The results for competing risk and logistic regression analysis were consistent with the conclusion from the main analysis (see supplementary table S3).

The interaction coefficient for duration of followup by drug use was statistically significant $(\mathrm{P}=0.008)$,

\begin{tabular}{|c|c|c|c|c|c|c|}
\hline \multirow[b]{2}{*}{ Characteristics } & \multicolumn{3}{|l|}{ Unadjusted } & \multicolumn{3}{|l|}{ Matched } \\
\hline & $\begin{array}{l}\text { Haloperidol } \\
(n=1668)\end{array}$ & $\begin{array}{l}\text { Atypical antipsychotics } \\
(n=4910)\end{array}$ & SD & $\begin{array}{l}\text { Haloperidol } \\
(n=1659)\end{array}$ & $\begin{array}{l}\text { Atypical antipsychotics } \\
(n=1659)\end{array}$ & SD \\
\hline \multicolumn{7}{|l|}{ Demographics: } \\
\hline Mean (SD) age (years) & $77.0(11.4)$ & $74.6(12.8)$ & 0.20 & $77.0(11.4)$ & $76.8(11.8)$ & 0.01 \\
\hline Women & $778(46.6)$ & $2316(47.2)$ & 0.01 & $773(46.6)$ & $802(48.3)$ & 0.04 \\
\hline White ethnicity & $1149(68.9)$ & $3586(73.0)$ & 0.09 & $1144(69.0)$ & $1154(69.6)$ & 0.01 \\
\hline Emergency room, or urgent admission & $1533(91.9)$ & $4624(94.2)$ & 0.09 & $1524(91.9)$ & $1522(91.7)$ & 0.00 \\
\hline \multicolumn{7}{|l|}{ Myocardial type: } \\
\hline NSTEMI & $1147(68.8)$ & $3299(67.2)$ & 0.02 & $1141(68.8)$ & $1130(68.1)$ & 0.02 \\
\hline STEMI & $441(26.4)$ & $1345(27.4)$ & 0.03 & $438(26.4)$ & $450(27.1)$ & 0.01 \\
\hline Unknown type & $80(4.8)$ & $266(5.4)$ & 0.03 & $80(4.8)$ & $79(4.8)$ & 0.00 \\
\hline \multicolumn{7}{|l|}{ Baseline comorbidity and treatments: } \\
\hline Mean (SD) Charlson comorbidity index score & $3.4(1.9)$ & $3.4(1.9)$ & 0.02 & $3.4(1.9)$ & $3.5(1.9)$ & 0.01 \\
\hline Heart failure & $953(57.1)$ & $2821(57.5)$ & 0.01 & $946(57.0)$ & $952(57.4)$ & 0.01 \\
\hline Dementia & $315(18.9)$ & $985(20.1)$ & 0.03 & $315(19.0)$ & $291(17.5)$ & 0.04 \\
\hline $\mathrm{PCl}$ or stent & $426(25.5)$ & $1243(25.3)$ & 0.01 & $422(25.4)$ & $415(25.0)$ & 0.01 \\
\hline ICU stay $\geq 1$ day & $1186(71.1)$ & $3527(71.8)$ & 0.02 & $1181(71.2)$ & $1162(70.0)$ & 0.03 \\
\hline Antiplatelet $s^{\star}$ & $1503(90.1)$ & $4244(86.4)$ & 0.11 & $1494(90.1)$ & $1493(90.0)$ & 0.00 \\
\hline Anticoagulantst & $1380(82.7)$ & $3908(79.6)$ & 0.08 & $1371(82.6)$ & $1361(82.0)$ & 0.02 \\
\hline Heparin, intravenous & $575(34.5)$ & $1695(34.5)$ & 0.00 & $570(34.4)$ & $564(34.0)$ & 0.01 \\
\hline Nitrates & $442(26.5)$ & $1241(25.3)$ & 0.03 & $440(26.5)$ & $443(26.7)$ & 0.00 \\
\hline Antiarrhythmics & $223(13.4)$ & $843(17.2)$ & 0.11 & $220(13.3)$ & $223(13.4)$ & 0.01 \\
\hline Benzodiazepines & $996(59.7)$ & 3137 (63.9) & 0.09 & $990(59.7)$ & $1021(61.5)$ & 0.04 \\
\hline Opioids & $1075(64.4)$ & $3113(63.4)$ & 0.02 & $1069(64.4)$ & $1106(66.7)$ & 0.05 \\
\hline \multicolumn{7}{|l|}{ Antipsychotic treatment: } \\
\hline Mean (SD) time to initiation & $5.3(4.8)$ & $5.6(6.5)$ & 0.05 & $5.3(4.8)$ & $5.4(5.6)$ & 0.02 \\
\hline Mean (SD) treatment duration (days) & $2.4(3.4)$ & $3.9(4.5)$ & 0.38 & $2.4(3.4)$ & $3.7(4.2)$ & 0.35 \\
\hline Drug switch during follow-up & $275(16.5)$ & $596(12.1)$ & 0.12 & $274(16.5)$ & $205(12.4)$ & 0.12 \\
\hline Discharged to SNF or hospice & $628(37.6)$ & $1829(37.3)$ & 0.01 & $651(39.2)$ & $625(37.7)$ & 0.03 \\
\hline Mean (SD) length of hospital stay (days) & $12.5(11.9)$ & $13.6(12.3)$ & 0.09 & $12.5(11.9)$ & $13.3(12.4)$ & 0.06 \\
\hline
\end{tabular}




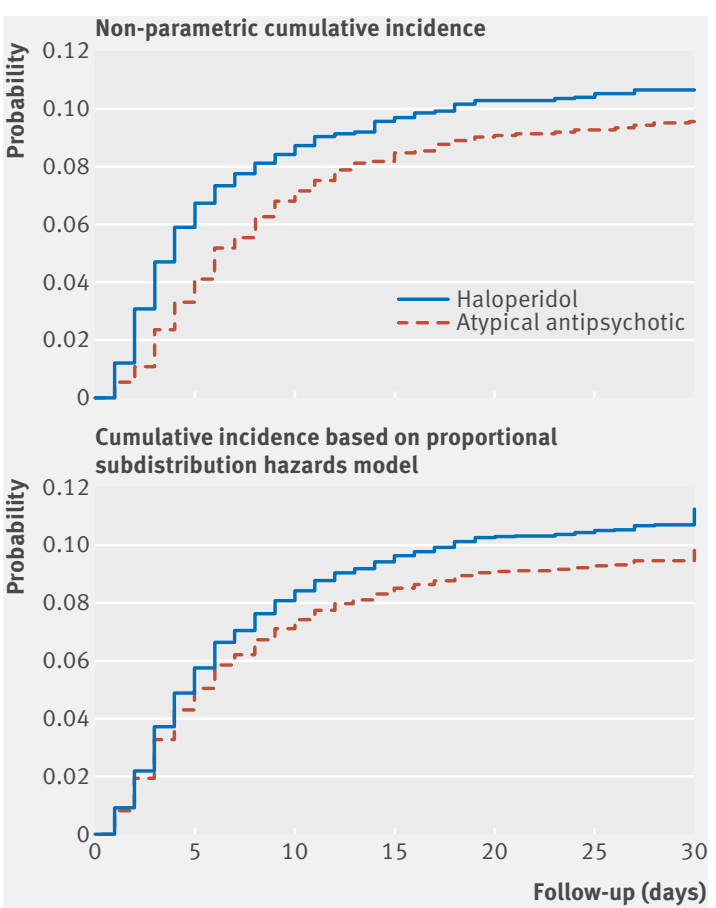

Fig 1 | Cumulative incidence function of in-hospital death in matched cohort during 30 days of follow-up, comparing haloperidol initiators to atypical antipsychotic initiators, accounting for hospital discharge as a competing risk

suggesting that the model does not meet the proportional hazards assumption. The adjusted hazard ratio was the largest on the day of antipsychotic initiation (3.28, 1.75 to 6.23 ) and decreased by $19 \%$ each day. By day 5 , an increased risk was no longer evident $(1.12,0.79$ to 1.59; see supplementary table S4).

The hazard ratios were similar across the subgroups based on age, although the absolute rate of death increased with advancing age (fig 2, see supplementary table S5). Using age or Charlson comorbidity index score as continuous variables in the model did not change the results. The hazard ratio was increased only among those with at least two days of treatment, but less so among those with one day of treatment only, although it was statistically non-significant. Comparison of hazard ratios between patients who were in the intensive care unit (hazard ratio 1.11, 0.68 to 1.81 ) on the index date and those who were on the medical ward $(2.01,1.44$ to 2.82$)$ suggests effect

\begin{tabular}{|c|c|c|c|c|c|}
\hline \multicolumn{6}{|c|}{$\begin{array}{l}\text { Table } 2 \text { | Hazard ratios of in-hospital dea } \\
\text { atypical antipsychotic initiators, based }\end{array}$} \\
\hline \multirow[b]{2}{*}{ Follow-up } & \multicolumn{2}{|c|}{ Haloperidol } & \multicolumn{2}{|c|}{ Atypical antipsychotics } & \multirow[b]{2}{*}{ Hazard ratio $(95 \% \mathrm{Cl})$} \\
\hline & No of deaths & Rate* $^{\star}$ & No of deaths & Rate & \\
\hline \multicolumn{6}{|l|}{ Unadjusted: } \\
\hline 7 days & 131 & 1.7 & 278 & 1.1 & $1.51(1.22$ to 1.85$)$ \\
\hline 30 days & 180 & 1.6 & 463 & 1.2 & 1.31 (1.11 to 1.56$)$ \\
\hline \multicolumn{6}{|l|}{ Matched: } \\
\hline 7 days & 129 & 1.7 & 92 & 1.1 & 1.50 (1.14 to 1.96$)$ \\
\hline 30 days & 178 & 1.6 & 159 & 1.3 & $1.26(1.01$ to 1.56$)$ \\
\hline
\end{tabular}

heterogeneity $(\mathrm{P}=0.04)$. Haloperidol was associated with an increased risk of death compared with each of olanzapine (adjusted hazard ratio 1.59, 1.13 to 2.24), quetiapine $(1.79,1.33$ to 2.41$)$, and risperidone (1.51, 1.12 to 2.03$)$.

Sensitivity analyses including patients who initiated treatment on day 1 or day 2, excluding patients with a recorded diagnosis of dementia, among patients with a recorded diagnosis of delirium, or using different modeling assumptions did not change the results (see supplementary table S6). In addition, in patients receiving haloperidol versus atypical antipsychotics we did not observe different trends in discontinuation of long term drugs or in use of opioids or benzodiazepines before antipsychotic initiation, proxies for terminal illness (see supplementary figure S3).

\section{Discussion}

In a large cohort of patients with acute myocardial infarction who were medically treated in hospital, initiation of oral haloperidol was associated with a small increase in the risk of in-hospital death compared with the initiation of oral atypical antipsychotics. The association appeared to be the strongest during the first four days after starting treatment. The results from sensitivity analyses were consistent with this main conclusion.

\section{Interpretation of study results}

The absolute difference in mortality during the seven days of follow-up (2.3\%) translates into a number needed to harm of 44 .

The study results were consistent across several analytic approaches, including the competing risk model and the logistic model analysis, which make different assumptions about the nature of the discharge event. Because the intention to treat analyses may be affected by exposure misclassification even during a relatively short follow-up period of seven days, we conducted as-treated analyses acknowledging that these are potentially subject to informative censoring and to time dependent confounding bias because treatment discontinuation or switching can be associated with a patient's prognosis. The slightly larger hazard ratios in as-treated analyses suggest that the potential adverse effect of haloperidol is more pronounced while patients are taking the drug compared with the time after they discontinue or switch. Given the decreasing hazard ratios over time in the intention to treat analysis, it is possible that selection of non-susceptible patients occurs in the underlying cohort over time. Alternatively, the decreasing hazard ratio over time might also indicate that haloperidol is associated with an increased risk shortly after initiation, but not later. This is supported by the fact that most ventricular arrhythmias occur in the first 48 hours after acute myocardial infarction, ${ }^{36}$ one of the suspected mechanisms by which haloperidol increases the risk of mortality. ${ }^{37}$ Results at later time points should be interpreted with caution, as only one third of the cohort is left in the risk set by day 7, and less than one quarter by day 10 . 


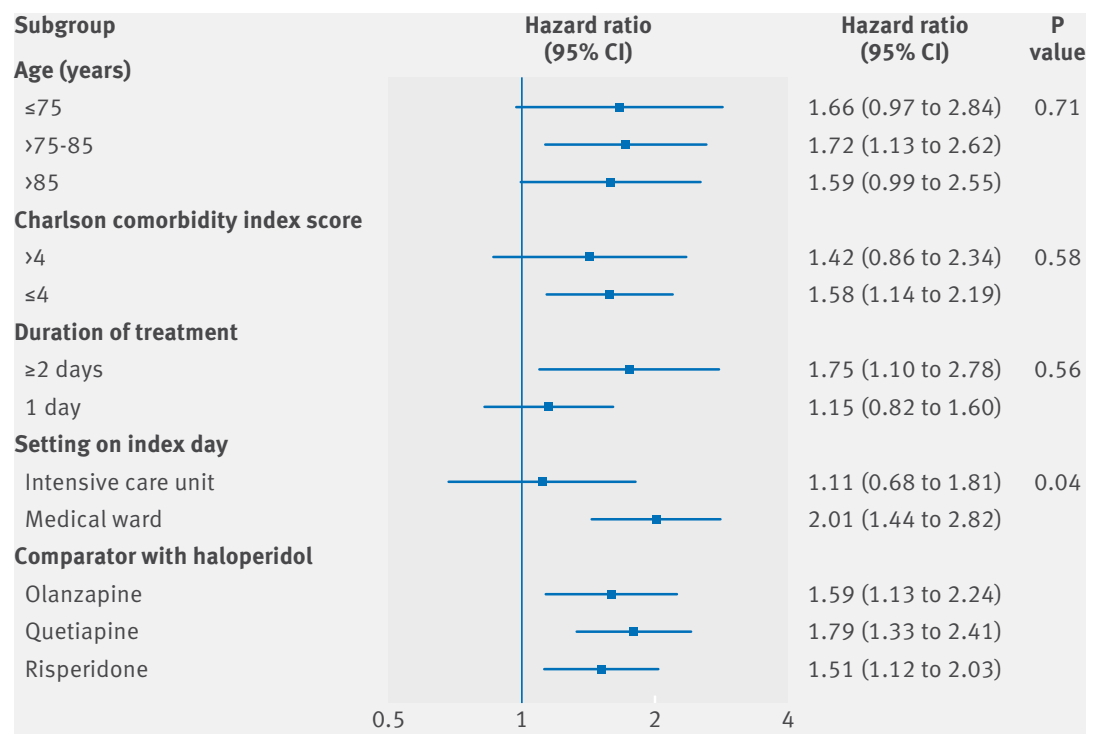

Fig 2 | Subgroup analyses comparing haloperidol initiators to atypical antipsychotic initiators, based on intention to treat analysis with seven days of follow-up

Comparison of the baseline characteristics in the unmatched cohort provided little evidence of confounding. We found that the association of haloperidol use and mortality became stronger after propensity score matching compared with the crude analysis in some analyses, suggesting negative confounding. ${ }^{38}$

There was some evidence of effect heterogeneity with an increased hazard ratio among patients who were in the medical ward on the index date, but less so for those who were in the intensive care unit. Patients in the intensive care unit are under a greater surveillance for any change in health status; so one possible explanation would be that potential adverse effects of haloperidol such as arrhythmia is more quickly taken care of in the intensive care unit and less likely to lead to death. Alternatively, we cannot exclude the possibility of confounding by terminal illness in the subgroup of patients in the medical ward.

\section{Relation to previous studies}

Owing to differences in the indications for antipsychotic use, evidence from outpatient studies might not be directly applicable to patients admitted to hospital. Moreover, the duration of antipsychotic treatment in hospital is short and treatment is often prescribed on an as necessary basis, ${ }^{39}$ unlike outpatient treatment, which is typically continued for several weeks to months. ${ }^{2}$ According to a recent review, ${ }^{19}$ six randomized trials compared use of typical antipsychotics with atypical antipsychotic and risk of mortality in patients admitted to hospital with delirium. ${ }^{15-184041}$ The largest treatment arm among these trials included 45 patients. The authors conducted a meta-analysis but did not find an association between any antipsychotic use and 30 day mortality (odds ratio 0.90, 95\% confidence interval 0.62 to 1.29$)$. However, this analysis combined delirium prevention trials with treatment trials because of limited sample size. Many of these trials took place in perioperative settings, which is different from this study's setting that excluded surgical patients. Another meta-analysis of trials comparing typical antipsychotics to placebo in elderly patients did not show a difference in mortality with a pooled sample of 2387 patients. ${ }^{20}$ But 14 out of 17 studies examined patients with dementia, whose antipsychotic use is different from that of patients with delirium. In the remaining three studies of patients with delirium, power was insufficient to determine whether there was a difference in mortality. Only two deaths occurred among 486 patients receiving placebo and none among 479 patients receiving typical antipsychotics. Two of the larger observational studies in patients admitted to hospital ( $n=2453$ and $n=244$ ) were not able to examine comparative safety because of no or too few deaths. ${ }^{2442}$ In addition, these studies were conducted in settings with heightened effort for detecting delirium and monitoring for adverse reactions, in contrast with the usual care setting in our study.

Cardiovascular death was suggested as the explanation for half of the excess deaths associated with typical antipsychotics in outpatients. ${ }^{37}$ Whether the cardiac side effects also explain the increased inhospital mortality remains unclear. The Cochrane review recommends using atypical antipsychotics rather than haloperidol if patients are likely to develop cardiac toxicity from the use of antipsychotics. ${ }^{39}$ While our study cannot address the reason for a potentially increased risk of death, atypical antipsychotics might be considered as a safer option in patients with substantial cardiac morbidity.

\section{Strengths and weaknesses of this study}

In this study we utilized a large nationwide hospital database, so the interpretation of the results is not limited to specific practices or hospitals. The large cohort size provided sufficient statistical power to detect a modest difference in mortality, which was not possible in earlier studies with a much smaller sample size. We were able to adjust for a large number of variables related to the treatments and procedures for each patient, which are measured without missingness, reducing the concern for residual confounding.

This study is not without limitations, however. We did not have information on patients before they were admitted to hospitals, so some comorbid conditions might be misclassified depending on the completeness of coding in the hospitals. It is possible that more diagnoses are recorded if a patient dies, which would affect the ability to control for confounding in our study. The true indication for antipsychotic use is unknown, and bias can be present if the true prevalence of delirium differed between haloperidol and atypical antipsychotics because patients without delirium are likely to have a lower risk of death compared with patients with delirium. However, a previous study has shown that the positive predictive value of antipsychotic use for delirium was $83 \%$ in patients admitted to hospital. ${ }^{43}$ Use of antipsychotics 
in hospital for the treatment of nausea has been reported, ${ }^{9}$ but such use would be infrequent in our study where we excluded patients who did not initiate oral antipsychotics and surgical patients. Also, the prevalence of recorded diagnoses of delirium based on ICD-9 codes was similar across the four antipsychotics examined in this study (19.6\% for haloperidol, $19.6 \%$ for olanzapine, $20.5 \%$ for quetiapine, and $18.5 \%$ for risperidone), and sensitivity analyses in this subset of patients showed an even stronger association, suggesting this is an unlikely explanation of the observed effect.

The duration of treatment was shorter for haloperidol than for atypical antipsychotics, which can lead to differential censoring bias. However, sensitivity analyses using a conditional model to account for this difference showed consistent, even stronger, effects. The perceived safety of atypical antipsychotics compared with haloperidol might be part of the reason why the treatment duration was shorter for haloperidol. ${ }^{9}$ Residual confounding can be an alternative explanation for the observed result, if the distributions of unmeasured factors such as disease severity, delirium subtype, or contraindications are imbalanced between the two groups. However, we did not find strong evidence of confounding based on close to 80 measured covariates even before matching, and it is unlikely that a few additional unmeasured variables can explain a 50\% increase in the risk independent of all other confounder and proxies of confounders that are adjusted for in our study. Similarly, it is possible that haloperidol is more often used in terminally ill patients as a comfort medication. ${ }^{33}$ But the trend of care characteristic of terminal illness before antipsychotic initiation such as opioid use or discontinuation of chronic disease care was similar between the two groups. Lastly, the mechanism of the increased risk of death is unknown since we did not have information on cause of death.

\section{Conclusions}

In a large, nationwide cohort of patients admitted to hospital, we found a small increase in the risk of death during the week after treatment initiation with haloperidol compared with atypical antipsychotics. The increased risk was strongest during the first four days and no longer evident by day 5 of follow-up. These findings are consistent with a higher risk of mortality associated with initiation of a typical versus atypical antipsychotics in the large body of evidence from previous studies in the outpatient setting. However, residual confounding cannot be completely excluded as a possible alternative explanation despite careful study design and adjustment for a wide range of potential confounders. In conclusion, although haloperidol has long been used to manage agitation or related symptoms for patients admitted to hospital, our findings suggest that atypical antipsychotics may be a less harmful option in older populations with acute myocardial infarction who require an off-label antipsychotic for severe agitation.
Contributors: YP designed the study, analyzed and interpreted data, and prepared the manuscript. BTB, DHK, SHD, EP, and KFH designed the study, interpreted data, and revised the manuscript for intellectual content. RJG interpreted data, gave advice on statistical analysis, and revised the manuscript for intellectual content. HM prepared data. The authors had full access to the data in the study. YP is the guarantor.

Funding: YP is funded by the Pharmacoepidemiology Program at Harvard TH Chan School of Public Health, which is supported by PhRMA Foundation. BTB is supported by the Eunice Kennedy Shriver National Institute of Child Health \& Human Development of the $\mathrm{NIH}$ (K08HD075831). DHK is supported by the Paul B Beeson clinical scientist development award in aging from the National Institute on Aging of the National Institutes of Health (NIH, K08AG051187). KFH is supported by the National Institute of Mental Health of the NIH (K01MH099141). The content is solely the responsibility of the authors and does not necessarily represent the official views of the $\mathrm{NIH}$.

Competing interests: All authors have completed the ICMJE uniform disclosure form at www.icmje.org/coi_disclosure.pdf and declare: DHK and BTB are consultants to the Alosa Foundation, a nonprofit educational organization with no relationship to any drug or device manufacturers; EP is consultant to Aetion; there are no other relationships or activities that could appear to have influenced the submitted work.

Ethical approval: The Partners institutional review board approved the use of the deidentified database for research.

Data sharing: No additional data available.

Transparency: The lead author (YP) affirms that this manuscript is an honest, accurate, and transparent account of the study being reported; that no important aspects of the study have been omitted; and that any discrepancies from the study as planned have been explained.

This is an Open Access article distributed in accordance with the Creative Commons Attribution Non Commercial (CC BY-NC 4.0) license, which permits others to distribute, remix, adapt, build upon this work non-commercially, and license their derivative works on different terms, provided the original work is properly cited and the use is noncommercial. See: http://creativecommons.org/licenses/by-nc/4.0/.

1 Huybrechts KF, Gerhard T, Crystal S, et al. Differential risk of death in older residents in nursing homes prescribed specific antipsychotic drugs: population based cohort study. BMJ 2012;344:e977. doi:10.1136/bmj.e977

2 Gerhard T, Huybrechts K, Olfson M, et al. Comparative mortality risks of antipsychotic medications in community-dwelling older adults. $\mathrm{Br}$ J Psychiatry 2014;205:44-51. doi:10.1192/bjp.bp.112.122499

3 Wang PS, Schneeweiss S, Avorn J, et al. Risk of death in elderly users of conventional vs. atypical antipsychotic medications. $\mathrm{N}$ EnglJ Med 2005;353:2335-41. doi:10.1056/NEJMoa052827

4 Schneeweiss S, Setoguchi S, Brookhart A, Dormuth C, Wang PS. Risk of death associated with the use of conventional versus atypical antipsychotic drugs among elderly patients. CMAI 2007;176: 627-32. doi:10.1503/cmaj.061250

5 Kales HC, Valenstein M, Kim HM, et al. Mortality risk in patients with dementia treated with antipsychotics versus other psychiatric medications. Am J Psychiatry 2007;164:1568-76, quiz 1623. doi:10.1176/appi.ajp.2007.06101710

6 Information for Healthcare Professionals. Conventional Antipsychotics 2008. FDA. http://www.Fda.Gov/Drugs/Drugsafety/ Postmarketdrugsafetyinformationforpatientsandproviders/ Ucm 124830. Htm Accessed November 1, 2013.

7 Public Health Advisory: Deaths with Antipsychotics in Elderly Patients with Behavioral Disturbances 2005. FDA. http://www.Fda.Gov/Drugs/ Drugsafety/Postmarketdrugsafetyinformationforpatientsandproviders/ Drugsafetyinformationforheathcareprofessionals/Publichealthadvisories/ Ucm053171.Htm Accessed November 1, 2013.

8 Park Y, Franklin JM, Schneeweiss S, et al. Antipsychotics and mortality: adjusting for mortality risk scores to address confounding by terminal illness. J Am Geriatr Soc 2015;63:516-23. doi:10.1111/jgs.13326

9 Herzig SJ, Rothberg MB, Guess JR, et al. Antipsychotic Use in Hospitalized Adults: Rates, Indications, and Predictors. J Am Geriatr Soc 2016:64:299-305. doi:10.1111/jgs.13943

10 Loh KP, Ramdass S, Garb JL, Brennan MJ, Lindenauer PK, Lagu T. From hospital to community: use of antipsychotics in hospitalized elders. $J$ Hosp Med 2014;9:802-4. doi:10.1002/jhm.2277

11 American Psychiatric Association. Practice Guideline for the Treatment of Patients with Delirium. American Psychiatric Association, 2010.

12 Barr J, Fraser GL, Puntillo K, et al, American College of Critical Care Medicine. Clinical practice guidelines for the management of pain, agitation, and delirium in adult patients in the intensive care unit. Crit Care Med 2013;41:263-306. doi:10.1097/ cCM.0b013e3182783b72 
13 American Geriatrics Society Expert Panel on Postoperative Delirium in Older Adults. Postoperative delirium in older adults: best practice statement from the American Geriatrics Society. J Am Coll Surg 2015;220:136-48.e1.

14 Girard TD, Pandharipande PP, Carson SS, et al, MIND Trial Investigators. Feasibility, efficacy, and safety of antipsychotics for intensive care unit delirium: the MIND randomized, placebocontrolled trial. Crit Care Med 2010;38:428-37. doi:10.1097/ cCM.0b013e3181c58715

15 Grover S, Kumar V, Chakrabarti S. Comparative efficacy study of haloperidol, olanzapine and risperidone in delirium. J Psychosom Res 2011;71:277-81. doi:10.1016/j. ipsychores.2011.01.019

16 Maneeton B, Maneeton N, Srisurapanont M, Chittawatanarat K. Quetiapine versus haloperidol in the treatment of delirium: a doubleblind, randomized, controlled trial. Drug Des Devel Ther 2013;7: 657-67. doi:10.2147/DDDT.S45575

17 Skrobik YK, Bergeron N, Dumont M, Gottfried SB. Olanzapine vs haloperidol: treating delirium in a critical care setting. Intensive Care Med 2004:30:444-9. doi:10.1007/s00134-003-2117-0

18 Han CS, Kim YK. A double-blind trial of risperidone and haloperidol for the treatment of delirium. Psychosomatics 2004;45:297-301. doi:10.1016/S0033-3182(04)70170-X

19 Neufeld KJ, Yue J, Robinson TN, Inouye SK, Needham DM Antipsychotic Medication for Prevention and Treatment of Delirium in Hospitalized Adults: A Systematic Review and Meta-Analysis. J Am Geriatr Soc 2016;64:705-14. doi:10.1111/jgs.14076

20 Hulshof TA, Zuidema SU, Ostelo RW, Luijendijk HJ. The Mortality Risk of Conventional Antipsychotics in Elderly Patients: A Systematic Review and Meta-analysis of Randomized Placebo-Controlled Trials. J Am Med Dir Assoc 2015;16:817-24. doi:10.1016/j. jamda.2015.03.015

21 Ozbolt LB, Paniagua MA, Kaiser RM. Atypical antipsychotics for the treatment of delirious elders. J Am Med Dir Assoc 2008;9:18-28. doi:10.1016/j.jamda.2007.08.007

22 Kim DH, Huybrechts KF, Patorno E, et al. Adverse Events Associated with Antipsychotic Use in Hospitalized Older Adults After Cardiac Surgery. J Am Geriatr Soc 2017;65:1229-37. doi:10.1111/ jgs.14768

23 Glassman AH, Bigger JT Jr. Antipsychotic drugs: prolonged QTc interval, torsade de pointes, and sudden death. Am J Psychiatry 2001;158:1774-82. doi:10.1176/appi. ajp.158.11.1774

24 Naksuk N, Thongprayoon C, Park JY, et al. Clinical Impact of Delirium and Antipsychotic Therapy: 10-Year Experience from a Referral Coronary Care Unit. Eur Heart J Acute Cardiovasc Care 2017;6:560-8

25 Premier Inc. About Premier Healthcare Database, 2016. http://www.Premierinc.Com/Transforming-Healthcare/HealthcarePerformance-Improvement/Premier-Research-Services/ Accessed September 2, 2016

26 Glare P, Miller J, Nikolova T, Tickoo R. Treating nausea and vomiting in palliative care: a review. Clin Interv Aging 2011;6:243-59. doi:10.2147/CIA.S13109

27 Inouye SK, Leo-Summers L, Zhang Y, Bogardus ST Jr, Leslie DL, Agostini JV. A chart-based method for identification of delirium: validation compared with interviewer ratings using the confusion assessment method. J Am Geriatr Soc 2005;53:312-8. doi:10.1111/ j.1532-5415.2005.53120.x

28 Swan JT, Fitousis K, Hall JB, Todd SR, Turner KL. Antipsychotic use and diagnosis of delirium in the intensive care unit. Crit Care 2012;16:R84. doi:10.1186/cc11342
29 Ahmed S, Leurent B, Sampson EL. Risk factors for inciden delirium among older people in acute hospital medical units: a systematic review and meta-analysis. Age Ageing 2014;43:326-33. doi:10.1093/ageing/afu022

30 Saczynski JS, Lessard D, Spencer FA, et al. Declining length of stay for patients hospitalized with AMI: impact on mortality and readmissions. Am J Med 2010;123:1007-15. doi:10.1016/j. amjmed.2010.05.018

31 Austin PC. An Introduction to Propensity Score Methods for Reducing the Effects of Confounding in Observational Studies. Multivariate Behav Res 2011;46:399-424. doi:10.1080/0027317 1.2011 .568786

32 Lee EW, Wei LJ, Amato DA, et al. Cox-Type Regression-Analysis for Large Numbers of Small-Groups of Correlated Failure Time Observations. Survival Analysis: State of the Art 1992;211:237-47.

33 Luijendijk HJ, de Bruin NC, Hulshof TA, Koolman X. Terminal illness and the increased mortality risk of conventional antipsychotics in observational studies: a systematic review. Pharmacoepidemiol Drug Saf 2016;25:113-22.

34 Caraceni A, Zecca E, Martini C, et al. Palliative sedation at the end of life at a tertiary cancer center. Support Care Cancer 2012;20: 1299-307. doi:10.1007/s00520-011-1217-6

35 Parsons C, Hughes CM, Passmore AP, Lapane KL. Withholding, discontinuing and withdrawing medications in dementia patients at the end of life: a neglected problem in the disadvantaged dying? Drugs Aging 2010;27:435-49. doi:10.2165/11536760000000000-00000

36 Mehta RH, Starr AZ, Lopes RD, et al, APEX AMI Investigators. Incidence of and outcomes associated with ventricular tachycardia or fibrillation in patients undergoing primary percutaneous coronary intervention. JAMA 2009;301:1779-89. doi:10.1001/ jama.2009.600

37 Setoguchi S, Wang PS, Alan Brookhart M, Canning CF, Kaci L, Schneeweiss S. Potential causes of higher mortality in elderly users of conventional and atypical antipsychotic medications. J Am Geriatr Soc 2008;56:1644-50. doi:10.1111/j.1532-5415.2008.01839.x

38 Mehio-Sibai A, Feinleib M, Sibai TA, Armenian HK. A positive or a negative confounding variable? A simple teaching aid for clinicians and students. Ann Epidemiol 2005;15:421-3. doi:10.1016/j. annepidem.2004.10.004

39 Lonergan E, Britton AM, Luxenberg J, Wyller T. Antipsychotics for delirium. Cochrane Database Syst Rev 2007;(2):CD005594.

40 Girard TD, Pandharipande PP, Carson SS, et al, MIND Trial Investigators. Feasibility, efficacy, and safety of antipsychotics for intensive care unit delirium: the MIND randomized, placebocontrolled trial. Crit Care Med 2010;38:428-37. doi:10.1097| CCM.0b013e3181c58715

41 Yoon HJ, Park KM, Choi WJ, et al. Efficacy and safety of haloperidol versus atypical antipsychotic medications in the treatment of delirium. BMC Psychiatry 2013;13:240. doi:10.1186/1471-244X$13-240$

42 Hatta K, Kishi Y, Wada K, et al. Antipsychotics for delirium in the general hospital setting in consecutive 2453 inpatients: a prospective observational study. Int J Geriatr Psychiatry 2014;29:253-62. doi:10.1002/gps.3999

$43 \mathrm{Kim} \mathrm{DH}$, Lee J, Kim CA, et al. Evaluation of algorithms to identify delirium in administrative claims and drug utilization database. Pharmacoepidemiol Drug Saf 2017;26:945-53. doi:10.1002/ pds. 4226

Supplementary information: web extra tables S1-6 and figures $\mathrm{S} 1-3$ 